Received: 07.11 .2018

Revised: 19.11 .2018

Accepted: 30.11 .2018

DOI: $10.17804 / 2410-9908.2018 .6 .100-116$

\title{
ON THE NUMERICAL-ANALYTICAL APPROACHES TO SOLVING A NONLINEAR HEAT CONDUCTION EQUATION WITH A SINGULARITY
}

\author{
A. L. Kazakov ${ }^{1,2, a)}$, L. F. Spevak ${ }^{1, b)}$, and O. A. Nefedova ${ }^{1, c^{*}}$ \\ ${ }^{1}$ Institute of Engineering Science, Ural Branch of the Russian Academy of Sciences, \\ 34 Komsomolskaya St., Ekaterinburg, 620049, Russian Federation \\ ${ }^{2}$ Matrosov Institute for System Dynamics and Control Theory, Siberian Branch of the Russian Academy of Sciences, \\ 134 Lermontova St., Irkutsk, 664033, Russian Federation

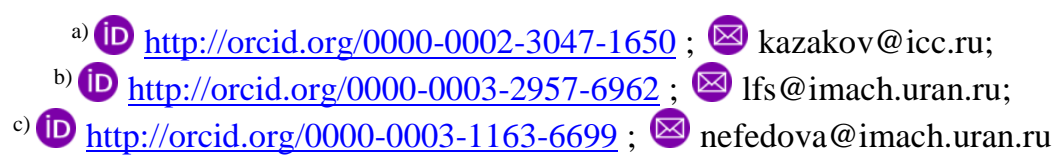 \\ *Corresponding author. E-mail: nefedova@imach.uran.ru \\ Address for correspondence: ul. Komsomolskaya, 34, 620049, Ekaterinburg, Russian Federation \\ Tel.: +7 (343) 36230 22; fax: +7 (343) 3745330
}

The paper deals with the construction and study of solutions to a nonlinear heat conduction equation in the case of the power-law dependence of the thermal conductivity coefficient on temperature. The parabolic type of the equation degenerates in the case of the zero value of the required function. It acquires some properties typical of first-order equations; particularly, it has solutions with a free boundary in the form of a heat wave propagating at a finite velocity over the cold front. Two types of the boundary conditions are discussed: the specified law of motion of the heat front and the boundary condition specified on a static manifold, initiating a heat wave. A comparative analysis of our developed approaches to solving the boundary value problems is made; namely, local analytical solutions are constructed by the power series method, and numerical-analytical solutions are constructed based on the boundary element method on a specified finite time interval. For some particular cases, the construction reduces to the Cauchy problem for a second-order ordinary nonlinear differential equation with a singularity before the higher derivative. The solution of this equation enables a partially self-similar solution to be constructed for the initial problem. The advantages and applicability of each approach are described. Examples are considered.

Keywords: nonlinear heat conduction equation, power series, boundary element method, partially self-similar solution.

\section{Acknowledgment}

The work was partially supported by the Complex Program of UB RAS (project No. 18-1-1-5) and the RFBR, project No. 16-01-00608.

\section{References}

1. Vazquez J.L. The Porous Medium Equation: Mathematical Theory. Oxford, Clarendon Press, 2007, 648 p. ISBN-10: 0198569033.

2. Samarskii A.A., Galaktionov V.A., Kurdyumov S.P., Mikhailov A.P. Blow-up in Quasilinear Parabolic Equations. NY, Berlin, Walter de Gruyte, 1995, 534 p.

3. Zeldovich Ya.B., Kompaneets A.S. On the theory of heat transfer with a temperaturedependent heat conductivity. In: Sbornik, posvyashchennyi 70-letiyu akademika A.F. Ioffe [Collec- 
tion in Honor of the 70th Birthday of Academician A.F. Ioffe]. Moscow, Izd. AN SSSR Publ., 1950, pp. 61-71. (In Russian).

4. Barenblatt G.I., Vishik I.M. On finite velocity of propagation of nonstationary filtration of a liquid or gas, Prikladnaya Matematika i Mekhanika, 1956, vol. 20, no. 3, pp. 411-417. (In Russian). 5. Oleinik O.A., Kalashnikov A.S. Chzhou Yui-Lin'. Cauchy problem and boundary problems for equations of nonstationary filtration type. Izv. Akad. Nauk SSSR. Ser. Mat., 1958, vol. 22, no. 5, pp. 667-704, (In Russian).

6. Sidorov A.F. In: Izbrannye Trudy: Matematika. Mekhanika [Selected Works: Mathematics. Mechanics]. Moscow, Fizmatlit Publ., 2001, 576 p. (In Russian). ISBN 5-9221-0103-X.

7. Polyanin A.D., Zaytsev V.F., Zhurov A.I. Nelineynye uravneniya matematicheskoy fiziki i mekhaniki. Metody resheniya [Nonlinear Equations in Mathematical Physics and Mechanics: Solution Methods]. Moscow, Yurayt Publ., 2017, 256 p. (In Russian). ISBN 978-5-534-02317-6.

8. Kazakov A.L., Lempert A.A. Analytical and Numerical Studies of the Boundary Value Problem of a Nonlinear Filtration with Degeneration. Vychislitelnye Tekhnologii, 2012, vol. 17, no. 1, pp. 57-68. (In Russian).

9. Kazakov A.L., Lempert A.A. Existence and Uniqueness of the Solution of the Boundary-Value Problem for a Parabolic Equation of Unsteady Filtration. Journal of Applied Mechanics and Technical Physic, 2013, vol. 54, iss. 2, pp. 251-258. Available at: https://doi.org/10.1134/S0021894413020107.

10. Kazakov A.L., Kuznetsov P.A. On the Analytic Solutions of a Special Boundary Value Problem for a Nonlinear Heat Equation in Polar Coordinates. Journal of Applied and Industrial Mathematics, 2018, vol. 12, iss. 2, pp. 255-263. Available at: https://doi.org/10.1134\%2FS1990478918020060.

11. Kazakov A.L., Kuznetsov P.A., Spevak L.F. On a Degenerate Boundary Value Problem for the Porous Medium Equation in Spherical Coordinates. Trudy Instituta Matematiki i Mekhaniki UrO RAN, 2014, vol. 20, no. 1, pp. 119-129. (In Russian).

12. Kazakov A.L., Spevak L.F. Boundary Elements Method and Power Series Method for One-dimensional Nonlinear Filtration Problems. Izvestiya IGU, Seriya "Matematika", 2012, vol. 5, no. 2, pp. 2-17. (In Russian).

13. Kazakov A.L., Spevak L.F. Numerical and analytical studies of a nonlinear parabolic equation with boundary conditions of a special form. Applied Mathematical Modelling, 2013, vol. 37, iss. 10-11, pp. 6918-6928. DOI: 10.1016/j.apm.2013.02.026.

14. Kazakov A.L., Spevak L.F. An analytical and numerical study of a nonlinear parabolic equation with degeneration for the cases of circular and spherical symmetry. Applied Mathematical Modelling, 2015, vol. 40, iss. 2, pp. 1333-1343. DOI: 10.1016/j.apm.2015.06.038.

15. Spevak L.F., Nefedova O.A. Solving a two-dimensional nonlinear heat conduction equation with degeneration by the boundary element method with the application of the dual reciprocity method. In: AIP Conference Proceedings, 2016, vol. 1785, iss. 1, pp. 040077. Available at: http://doi.org/10.1063/1.4967134.

16. Kazakov A.L., Spevak L.F., Nefedova O.A. Solution of a two-dimensionel problem on the motion of a heat wave front with the use of power series and the boundary element method. Izvestiya IGU, Seriya "Matematika”, 2016, vol. 18, pp. 21-37. (In Russian).

17. Banerjee P.K., Butterheld R. Boundary element methods in engineering science. US, McGraw-Hill Inc., 1981, 452 p. ISBN-10: 0070841209, ISBN-13: 978-0070841208.

18. Brebbia C.A., Telles J.F.C., Wrobel L.C. Boundary Element Techniques. Berlin, Neidelberg, New York, Tokyo, Springer-Verlag, 1984, 466 p. ISBN: 978-3-642-48862-7. DOI: 10.1007/978-3-642-48860-3.

19. Nardini D., Brebbia C.A. A New Approach to Free Vibration Analysis using Boundary Elements. Applied Mathematical Modelling, 1983, vol. 7, no. 3, pp. 157-162. DOI: 10.1016/0307904X(83)90003-3.

20. Fedotov V.P., Spevak L.F. One approach to the derivation of exact integration formulae in the boundary element method. Engineering Analysis with Boundary Elements, 2008, vol. 32, no. 10, pp. 883-888. DOI: $10.1016 /$ j.enganabound.2008.03.001 
Подана в журнал: 07.11.2018

УДК 517.958:519.633

DOI: $10.17804 / 2410-9908.2018 .6 .100-116$

\title{
О ЧИСЛЕННО-АНАЛИТИЧЕСКИХ ПОДХОДАХ К РЕШЕНИЮ НЕЛИНЕЙНОГО УРАВНЕНИЯ ТЕПЛОПРОВОДНОСТИ С ОСОБЕННОСТЬЮ
}

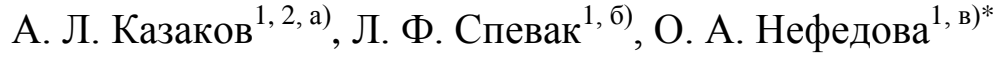 \\ ${ }^{1}$ Федеральное государственное бюджетное учреждение науки \\ Институт машиноведения Уральского отделения Российской академии наук, \\ 620049, ул. Комсомольская, 34, Екатеринбург, Российская Федерация \\ ${ }^{2}$ Федеральное государственное бюджетное учреждение науки Институт динамики систем и теории \\ управления имени В.М. Матросова Сибирского отделения Российской академии наук, \\ 664033, ул. Лермонтова, 134, Иркутск, Российская Федерации

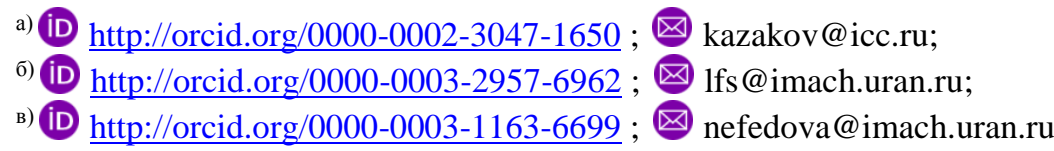 \\ *Ответственный автор. Электронная почта: nefedova@imach.uran.ru \\ Адрес для переписки: ул. Комсомольская, 34, 620049, Екатеринбург, Российская Федерация \\ Тел.: +7 (343) 362-30-22; факс: +7 (343) 374-53-30
}

Работа посвящена построению и исследованию решений нелинейного уравнения теплопроводности в случай степенной зависимости коэффициента теплопроводности от температуры. Параболический тип рассматриваемого уравнения вырождается в случае нулевого значения искомой функции. При этом оно приобретает некоторые свойства, характерные для уравнений первого порядка, в частности, обладает решениями со свободной границей типа тепловой волны, распространяющейся по холодному фону с конечной скоростью. Рассматриваются два типа краевых условий: заданный закон движения теплового фронта; краевой режим, заданный на неподвижном многообразии, инициирующий тепловую волну. Проводится сравнительный анализ подходов к решению указанных краевых задач, разработанных авторами: методом степенных рядов строятся локально-аналитические решения; на основе метода граничных элементов на заданном конечном промежутке времени строятся численноаналитические решения. При этом для отдельных частных случаев построение сводится к задаче Коши для обыкновенного нелинейного дифференциального уравнения второго порядка с особенностью перед старшей производной. Решение этого уравнения позволяет построить для исходной задачи частично-автомодельное решение. Описаны преимущества и области применимости каждого из подходов. Рассмотрены примеры.

Ключевые слова: нелинейное уравнение теплопроводности, степенной ряд, метод граничных элементов, частично-автомодельное решение.

\section{1. Введение}

В статье обобщен накопленный к настоящему моменту опыт авторов по решению нелинейного параболического уравнения теплопроводности. Мы попытались определить место каждого из разработанных подходов, сопоставить полученные решения и определить возможные пути развития.

Рассмотрим нелинейное уравнение теплопроводности для случая степенной зависимости коэффициента теплопроводности от температуры $[1,2]$ 


$$
T_{t}=\alpha \operatorname{div}\left(T^{\sigma} \nabla T\right)
$$

Здесь $t$ - время; $T$ - искомая функция (температура); $\alpha, \sigma$ - положительные константы; div, $\nabla$ - дивергенция и градиент по пространственным координатам. Уравнение (1.1) стандартной подстановкой $u=T^{\sigma}, t^{\prime}=\alpha t$ сводится к уравнению

$$
u_{t}=u \Delta u+\frac{1}{\sigma}(\nabla u)^{2}
$$

Одним из важных и интересных видов решений уравнения (1.2) являются решения типа тепловой волны, которая распространяется по холодному (нулевому) фону с конечной скоростью. Первые примеры такого рода решений были, насколько известно авторам, предложены в статье Я.Б. Зельдовича и А.С. Компанейца [3]. В вышедших несколько позднее работах Г.И. Баренблатта [4] и О.А. Олейник [5] приведены решения (1.2) типа волн фильтрации. В классе аналитических функций последние исследовали А.Ф. Сидоров [6] и его ученики. Обзор полученных упомянутыми и некоторыми другими учеными результатов можно найти в [7].

Авторы занимаются построением решений типа тепловой волны для уравнения (1.2) с 2012 г., продолжая и развивая результаты А.Ф. Сидорова. В работах [8, 9] рассматривался одномерный случай, статья [10] посвящена двумерному, а [11] - трехмерному случаям. Также к 2012 г. относится первый опыт применения авторами метода граничных элементов (МГЭ) к построению решений типа тепловой волны с заданным фронтом для уравнения (1.2) в плоскосимметричном случае [12], при этом для верификации результатов расчетов использованы отрезки степенных рядов. Позднее разработанный подход к решению с помощью МГЭ был распространен на случаи других краевых режимов [13], а также на задачи с круговой и сферической симметриями [14]. Далее были разработаны алгоритмы решения двумерных задач $[15,16]$.

Важным вопросом при проведении численных расчетов является верификация их результатов. Хорошим инструментом для этого является сравнение с точными решениями рассматриваемого уравнения. Некоторые из них можно найти в [2, 7].

Настоящая статья в основном носит обзорный характер. В ней проводится сравнительный анализ разработанных авторами подходов к исследованию краевых задач, имеющих решения типа тепловой волны: методом степенных рядов строятся аналитические решения в малой окрестности начального момента времени; на основе метода граничных элементов на заданном конечном промежутке времени строятся численно-аналитические решения. Описаны преимущества и области применимости каждого из подходов. Также приводятся и некоторые новые, ранее неопубликованные результаты. Так, для задачи о движении тепловой волны с заданным фронтом строятся точные (частично-автомодельные) решения. При этом построение сводится к задаче Коши для обыкновенного нелинейного дифференциального уравнения второго порядка с особенностью перед старшей производной. Проводятся численные расчеты, для верификации результатов которых используются найденные точные решения.

\section{2. Постановка краевых задач}

В одномерном случае уравнение (1.2) имеет вид

$$
u_{t}=u u_{\rho \rho}+\frac{1}{\sigma} u_{\rho}^{2}+\frac{v}{\rho} u u_{\rho}
$$


Здесь $\rho$ - пространственная координата; параметр $v$ принимает значения $0 ; 1 ; 2$ для задач теплопроводности на прямой, на плоскости (задачи с круговой симметрией) и в пространстве (задачи со сферической симметрией) соответственно.

Уравнение (2.1) имеет решение типа тепловой волны при каждом из следующих краевых условий:

$$
\begin{gathered}
\left.u\right|_{\rho=a(t)}=0, \\
\left.u\right|_{\rho=R}=f(t) .
\end{gathered}
$$

Здесь $a^{\prime}(0)>0 ; f(0)=0 ; f^{\prime}(0)>0 ;$ при $v=0 \quad a(0)=0 ; R=0$, при $v=1,2 a(0)>0$; $R>0$. Задача (2.1), (2.2) называется задачей о движении теплового фронта. Задача (2.1) и (2.3) является задачей об инициировании тепловой волны.

В двумерном случае уравнение (1.2) имеет вид

$$
u_{t}=u\left(u_{x_{1} x_{1}}+u_{x_{2} x_{2}}\right)+\frac{1}{\sigma}\left(u_{x_{1}}^{2}+u_{x_{2}}^{2}\right) .
$$

Здесь $x_{1}, x_{2}-$ пространственные координаты. Закон движения фронта тепловой волны, аналогичный (2.2), задается краевым условием

$$
\left.u\right|_{b\left(t, x_{1}, x_{2}\right)=0}=0
$$

где уравнение $b\left(t, x_{1}, x_{2}\right)=0$ в каждый момент времени определяет фронт тепловой волны $S^{(t)}$ - замкнутую гладкую линию, ограничивающую область $V^{(t)}$, содержащую начало координат. Предполагается, что если $t_{1}<t_{2}$, то $V^{\left(t_{1}\right)} \subset V^{\left(t_{2}\right)}$. Задача о движении тепловой волны состоит в определении функции $u=u\left(t, x_{1}, x_{2}\right)$ в области $t \in\left[0, t_{*}\right], x\left(x_{1}, x_{2}\right) \in \Omega^{(t)}$, где $\Omega^{(t)}-$ область, ограниченная границами $S^{(0)}$ и $S^{(t)}$.

Задача об инициировании тепловой волны также определяется краевым условием, заданным на замкнутой линии:

$$
\left.u\right|_{a\left(x_{1}, x_{2}\right)=0}=f\left(t, x_{1}, x_{2}\right) .
$$

Здесь уравнение $a\left(x_{1}, x_{2}\right)=0$ задает замкнутую гладкую линию, ограничивающую область, содержащую начало координат, $f\left(0, x_{1}, x_{2}\right)=0 ; f_{t}\left(0, x_{1}, x_{2}\right)>0$.

В полярных координатах уравнения (2.4)-(2.6) имеют следующий вид:

$$
\begin{gathered}
u_{t}=u u_{\rho \rho}+\frac{u_{\rho}^{2}}{\sigma}+\frac{u u_{\rho}}{\rho}+\frac{1}{\rho^{2}}\left(\frac{u_{\varphi}^{2}}{\sigma}+u u_{\varphi \varphi}\right) \\
\left.u\right|_{\rho=b(t, \varphi)}=0 \\
\left.u\right|_{\rho=R(\varphi)}=f(t, \varphi) .
\end{gathered}
$$


Отметим, что для всех поставленных выше задач характерно общее свойство: на фронте тепловой волны обращается в нуль множитель перед второй производной по координате $\rho$, что приводит к вырождению параболического типа уравнения.

\section{3. Аналитическое решение методом степенных рядов}

Построение решений в виде специальных рядов является одним из основных методов исследования краевых задач математической физики с вырождением, применяемых в научной школе ак. А.Ф. Сидорова [6], к которой принадлежит один из авторов статьи.

Запишем краевые условия для уравнения (2.1) в виде

$$
\left.u\right|_{\rho=a(t)}=f(t) .
$$

Легко видеть, что (2.2) и (2.3) являются частными случаями (3.1). Будем предполагать, что

$$
a(0)>0, f(0)=0, f^{\prime}(0) \geq 0,\left[a^{\prime}(0)\right]^{2}+\left[f^{\prime}(0)\right]^{2}>0 .
$$

Кроме того, пусть функции $a(t), f(t)$ являются аналитическими, т. е. совпадают в некоторой окрестности $a(t)$ со своим тейлоровским разложением:

$$
a(t)=\sum_{k=0}^{\infty} \frac{a_{k}}{k !} t^{k}, a_{k}=\left.\frac{d^{k} a(t)}{d t^{k}}\right|_{t=0} ; f(t)=\sum_{k=0}^{\infty} \frac{f_{k}}{k !} t^{k}, f_{k}=\left.\frac{d^{k} f(t)}{d t^{k}}\right|_{t=0}
$$

Решение задачи (2.1) и (3.1) строится в виде кратного степенного ряда:

$$
u=\sum_{k, l=0}^{\infty} \frac{u_{k, l} t^{k} r^{l}}{k ! l !}, u_{k, l}=\left.\frac{\partial^{k+l} u}{\partial t^{k} \partial r}\right|_{t=0, r=0}
$$

Здесь $r=\rho-a(t)$. При этом из (3.1) имеем, что $u_{k, 0}=f_{k}$, в том числе $u_{0,0}=f_{0}=0$; $u_{1,0}=f_{1}$. Для нахождения $u_{0,1}$ положим в уравнении (2.1) $t=r=0$. С учетом (3.2) получаем квадратное уравнение $f_{1}=u_{0,1}^{2} / \sigma+a_{1} u_{0,1}$, имеющее два действительных корня

$$
u_{0,1}=-\frac{\sigma a_{1}}{2} \pm \sqrt{\left(\frac{\sigma a_{1}}{2}\right)^{2}+\sigma f_{1}}
$$

Выбор знака перед корнем соответствует выбору направления движения тепловой волны (к началу координат или от него). Дальнейшее построение коэффициентов ряда (3.4) производится индукцией по суммарному порядку дифференцирования $n=k+l$. При этом, как легко убедиться, база индукции уже сформирована.

Пусть найдены коэффициенты до порядка $n$ включительно. Тогда, чтобы найти коэффициенты порядка $n+1$, продифференцируем уравнение (2.1) $k$ раз по $t, n-k$ раз по $r$, изменяя $k$ от нуля до $n$, и положим $t=r=0$. Получим трехдиагональную систему линейных алгебраических уравнений (СЛАУ) порядка $n$. Вид системы здесь не приводится из-за громоздкости [14]. Отметим здесь лишь, что хотя условие диагонального преобладания для упомянутой СЛАУ, вообще говоря, не выполняется, она является крамеровской, что обеспечивает однозначную определимость коэффициентов ряда (3.4). 
Двумерный случай рассматривается аналогично. Краевые условия для уравнения (2.7) записываются в виде

$$
\left.u\right|_{\rho=b(t, \varphi)}=f(t, \varphi),
$$

который обобщает (2.8) и (2.9). Решение задачи (2.7) и (3.6) строится в виде ряда (3.4) с коэффициентами, которые определяются на первом шаге при решении квадратного уравнения, а на последующих - при решении невырожденных трехдиагональных СЛАУ, с той разницей, что их значения будут уже не константами, а функциями переменной $\varphi$.

Отметим, что сходимость построенных рядов обеспечивают ранее доказанные утверждения. Так, авторами ранее были обоснованы теоремы существования и единственности аналитических решений в одномерном[8,9] и двумерном [10] случаях.

Метод степенных рядов позволяет построить в области сходимости последних решений с любой наперед заданной точностью. Однако сходятся ряды, как правило, в малой окрестности начального момента времени $t=0$, причем оценить ее снизу удается только в некоторых частных случаях. Указанное обстоятельство вынуждает при проведении численных расчетов искать альтернативные подходы, одним из которых является применение метода граничных элементов.

\section{4. Алгоритмы решения методом граничных элементов}

Стандартный подход к решению методом граничных элементов линейных уравнений параболического типа предполагает использование фундаментального решения параболического сингулярного уравнения, зависящего от времени $[17,18]$. Нелинейность и вырождающийся тип уравнения (1.2) делают такой подход невозможным. Еще одним фактором, затрудняющим стандартный подход к решению, является изменяющаяся во времени область решения краевой задачи - область ненулевых значений искомой функции $u$. В связи с этим в работах [11-16] авторами был предложен следующий подход к применению МГЭ для решения краевых задач, поставленных в п. 2.

Задачи решаются по шагам по времени. На каждом шаге $t=t_{k}$ уравнение (1.2) представляется в виде уравнения Пуассона:

$$
\Delta u=\frac{1}{u}\left(u_{t}-\frac{(\nabla u)^{2}}{\sigma}\right) .
$$

\section{1. Применение МГЭ для решения одномерных задач}

В одномерных случаях на шаге $t=t_{k}$ решается краевая задача в области $\Omega_{k}: \rho \in[R, L]$, $L=a\left(t_{k}\right)$. Для задачи (2.1) и (2.2) область $\Omega_{k}$ является известной, уравнение (4.1) решается при граничных условиях

$$
\begin{gathered}
\left.u\right|_{\rho=L}=0 ; \\
\left.q\right|_{\rho=L}=-\sigma a^{\prime}\left(t_{k}\right) .
\end{gathered}
$$


Здесь $q=\frac{\partial u}{\partial n}-$ поток; $n$ - внешняя нормаль к границе области решения задачи, $n(R)=-1 ; n(L)=1 ;$ условие (4.3) выполняется для решения уравнения (4.1) вдоль нулевого фронта тепловой волны.

Для задачи (2.1) и (2.3) параметр $L$, а следовательно, и область $\Omega_{k}$, не заданы краевым условием (фронт тепловой волны неизвестен). В этом случае уравнение (4.1) решается при граничном условии

$$
\left.u\right|_{\rho=R}=f\left(t_{k}\right)
$$

и условиях (4.2) и (4.3) на неизвестном тепловом фронте. При этом значение $L=a\left(t_{k}\right)$ (фронт волны) определяется в процессе решения.

Решение задач в плоскосимметричном случае ( $v=0$ в уравнении (2.1)) строится с использованием фундаментального решения для одномерных задач эллиптического типа [17]. Поскольку правая часть уравнения (4.1) зависит от искомой функции, решение находится итерационно. Итерационная процедура для задачи (4.1)-(4.3) при заданном фронте волны выглядит следующим образом:

$$
u^{(n)}(\xi)=q_{1}^{(n)} u^{*}(\xi, R)-\sigma a^{\prime}\left(t_{k}\right) u^{*}(\xi, L)-u_{1}^{(n)} q^{*}(\xi, R)-\int_{R}^{L} \frac{1}{u^{(n)}}\left(u_{t}^{(n)}-\frac{\left(u_{\rho}^{(n)}\right)^{2}}{\sigma}\right) u^{*}(\xi, x) d x,
$$

где $u^{*}(\xi, x)$ - фундаментальное решение для одномерных задач [17]; $q^{*}(\xi, x)=\frac{\partial u^{*}(\xi, x)}{\partial n}$; $u^{(n)}(\xi)-n$-я итерация решения; $u^{(0)}(\xi) \equiv 0, u_{1}^{(n)}=u^{(n)}(L) ; q_{1}^{(n)}=\left.\frac{\partial u^{(n)}}{\partial n}\right|_{\xi=L}-n$-е итерации граничных значений искомой функции и потока, определяемые из системы граничных интегральных уравнений [17].

Процедура решения для задачи об инициации тепловой волны, (4.1)-(4.4), отличается добавлением к системе граничных интегральных уравнений разностной аппроксимации условия (4.3) для определения дополнительной неизвестной $L[12,13]$.

Решение задачи в случае круговой симметрии $(v=1)$ строится с использованием фундаментального решения для двумерных задач теории потенциала [18]. Итерационное решение методом граничных элементов двумерной задачи (4.1)-(4.3) с круговой симметрией на шаге $t=t_{k}$ сводится к процедуре

$$
u^{(n)}\left(\rho_{\xi}\right)=q_{1}^{(n)} \bar{u}^{*}\left(\rho_{\xi}, R\right)-\sigma a^{\prime}\left(t_{k}\right) \bar{u}^{*}\left(\rho_{\xi}, L\right)-u_{1}^{(n)} \bar{q}^{*}\left(\rho_{\xi}, R\right)-\int_{R}^{L} \frac{1}{u^{(n)}}\left(u_{t}^{(n)}-\frac{\left(u_{\rho}^{(n)}\right)^{2}}{\sigma}\right) \bar{u}^{*}\left(\rho_{\xi}, \rho_{x}\right) d x
$$

где $\rho_{\xi}, \varphi_{\xi}$ - полярные координаты точки $\xi ; \rho_{x}, \varphi_{x}-$ полярные координаты точки $x$; $\bar{u}^{*}\left(\rho_{\xi}, \rho_{x}\right)=\rho_{x} \int_{0}^{2 \pi} u^{*}(\xi, x) d \varphi_{x} ; \quad \bar{q}^{*}\left(\rho_{\xi}, \rho_{x}\right)=\rho_{x} \int_{0}^{2 \pi} q^{*}(\xi, x) d \varphi_{x}-$ специальное фундаментальное решение для случая круговой симметрии; $u^{*}(\xi, x)$ - фундаментальное решение для 
двумерных задач [18]; $q^{*}(\xi, x)=\frac{\partial u^{*}(\xi, x)}{\partial n}$. Специальное фундаментальное решение было найдено в аналитическом виде:

$$
\bar{u}^{*}\left(\rho_{\xi}, \rho_{x}\right)=\left\{\begin{array}{l}
-\rho_{x} \ln \rho_{x}, \rho_{\xi} \leq \rho_{x} \\
-\rho_{x} \ln \rho_{\xi}, \rho_{\xi}>\rho_{x}
\end{array} .\right.
$$

Решение задачи о движении теплового фронта (4.1)-(4.3) на шаге $t=t_{k}$ в случае сферической симметрии $(v=2)$ сводится к итерационной процедуре (4.6), в которой $\bar{u}^{*}\left(\rho_{\xi}, \rho_{x}\right)=\rho_{x} \int_{0}^{\pi} \int_{0}^{2 \pi} u^{*}(\xi, x) d \varphi_{x} d \vartheta_{x} ; \bar{q}^{*}\left(\rho_{\xi}, \rho_{x}\right)=\int_{0}^{\pi} \int_{0}^{2 \pi} q^{*}(\xi, x) d \varphi_{x} d \vartheta_{x} ; \rho, \varphi, \vartheta-$ сферические координаты; $u^{*}(\xi, x)$ - фундаментальное решение для трехмерных задач $[18] ; q^{*}(\xi, x)=\frac{\partial u^{*}(\xi, x)}{\partial n}$. Специальное фундаментальное решение для этого случая также было найдено в аналитическом виде:

$$
\bar{u}^{*}\left(\rho_{\xi}, \rho_{x}\right)=\left\{\begin{array}{l}
\rho_{x}, \rho_{\xi} \leq \rho_{x} \\
\frac{\rho_{x}^{2}}{\rho_{\xi}}, \rho_{\xi}>\rho_{x}
\end{array} .\right.
$$

Решение задачи об инициации тепловой волны, (4.1)-(4.4), в случаях $v=1,2$, аналогично плоскосимметричному случаю, отличается добавлением к системе граничных интегральных уравнений разностной аппроксимации условия (4.3) для определения дополнительной неизвестной $L$.

Отметим, что интегралы по области решения задачи, входящие в (4.5) и (4.6), вычисляются с использованием метода двойственной взаимности [19].

Построенные алгоритмы позволяют найти для каждого значения времени $t=t_{k}$ непрерывные по пространственной переменной решения исходных задач о движении теплового фронта и об инициировании тепловой волны. С помощью разработанных алгоритмов было решено большое количество задач. Сравнение построенных решений с известными точными решениями дало хорошие результаты. Стоит отметить при этом, что точность решения задач о движении теплового фронта была несколько выше, чем для задач об инициировании волны. Кроме того, существенное отличие при решении двух типов задач было отмечено относительно сходимости итерационных процедур. При решении задач о движении теплового фронта наблюдалась устойчивая хорошая сходимость, в то время как итерационные процедуры для задач об инициации волны сходились нестабильно и не для всех граничных условий. Это объясняется, безусловно, тем, что область решения задачи об инициировании волны на каждом шаге неизвестна.

\section{2. Применение МГЭ для решения двумерных задач}

Пошаговое решение двумерной задачи о движении теплового фронта (2.4) и (2.5) производится следующим образом. На шаге $t=t_{k}$ в области $\Omega^{\left(t_{k}\right)}$ рассматривается уравнение Пуассона (4.1) с граничными условиями, вытекающими из условий (2.5): 


$$
\begin{gathered}
\left.u\right|_{x \in S}\left(t_{k}\right)=0, \\
\left.q\right|_{x \in S}\left(t_{k}\right)=\frac{\sigma b_{t}}{\sqrt{b_{x_{1}}^{2}+b_{x_{2}}^{2}}} .
\end{gathered}
$$

Итерационное решение задачи (4.1), (4.9) и (4.10) методом граничных элементов приводит к соотношению

$$
\begin{gathered}
u^{(n)}(\xi)=\sum_{j=1}^{N}\left(q_{j}^{(n)} \int_{e_{j}} u^{*}\left(\xi_{i}, x\right) d x-u_{j}^{(n)} \int_{e_{j}} q^{*}\left(\xi_{i}, x\right) d x\right)- \\
-\int_{\Omega^{\left(t_{k}\right)}} \frac{1}{u^{(n-1)}}\left(u_{t}^{(n-1)}-\frac{\left(u_{x_{1}}^{(n-1)}\right)^{2}+\left(u_{x_{2}}^{(n-1)}\right)^{2}}{\sigma}\right) u^{*}(\xi, x) d \Omega(x),
\end{gathered}
$$

где $u^{*}(\xi, x) ; q^{*}(\xi, x)$ соответствуют двумерным задачам [18] $e_{i} ; i=1, \ldots, N,-$ граничные элементы с постоянной аппроксимацией, на которые разбита граница области $\Omega^{\left(t_{k}\right)}, \xi_{i}-$ соответствующие им узлы, $u_{i}^{(n)} ; q_{i}^{(n)}-n$-е итерации узловых значений искомой функции и потока, которые на каждой итерации определяются из решения системы граничных интегральных уравнений:

$$
\begin{gathered}
\frac{1}{2} u_{i}=\sum_{j=1}^{N}\left(q_{j} \int_{e_{j}} u^{*}\left(\xi_{i}, x\right) d x-u_{j} \int_{e_{j}} q^{*}\left(\xi_{i}, x\right) d x\right)- \\
-\int_{\Omega^{\left(t_{k}\right)}} \frac{1}{u^{(n-1)}}\left(u_{t}^{(n-1)}-\frac{\left(u_{x_{1}}^{(n-1)}\right)^{2}+\left(u_{x_{2}}^{(n-1)}\right)^{2}}{\sigma}\right) u^{*}\left(\xi_{i}, x\right) d \Omega(x), i=1, \ldots, N .
\end{gathered}
$$

Отметим, что незвестными в системе (4.12) являются лишь значения искомой функции и потока на элементах, не заданные граничными условиями (4.9) и (4.10), т. е. на элементах границы $S^{(0)}$. Отсюда следует необходимость соблюдать равенство числа элементов, лежащих на $S^{(0)}$, числу элементов на $S^{\left(t_{k}\right)}$. Все интегралы по граничным элементам вычисляются с помощью точных аналитических формул [20]. Интегралы по области $\Omega^{\left(t_{k}\right)}$, входящие в соотношения (4.11) и (4.12), сводятся к интегралам по ее границе с помощью метода двойственной взаимности [19].

Представленный алгоритм позволяет построить на каждом шаге по времени $t=t_{k}$ непрерывное по пространственным координатам решение исходной задачи (2.4) и (2.5). Алгоритм был реализован для большого количества задач $[15,16]$. Сравнение полученных решений с известными точными решениями показало высокую точность расчетов.

Решение задачи об инициировании тепловой волны (2.4) и (2.6) аналогичным образом невозможно, поскольку нулевой фронт $S^{\left(t_{k}\right)}$ в каждый момент времени неизвестен, и определить его в процессе решения не представляется возможным ввиду необходимости 
вычисления интегралов по неизвестной области $\Omega^{\left(t_{k}\right)}$ и неизвестным граничным элементам. Для решения двумерных задач об инициировании тепловой волны, а также для улучшения сходимости итерационных процедур при решении одномерных задач об инициировании тепловой волны, была использована специальная замена переменных, описанная в следующем разделе.

\section{5. Замена переменных для решения задач об инициировании волны}

Для решения одномерной задачи об инициировании тепловой волны поменяем местами искомую функцию $u$ и пространственную переменную $\rho$ в уравнении (2.1). Подобная замена в уравнении ранее использовалась в работах А.Ф. Сидорова [6]. Замена будет корректной в случае монотонной функции $f(t)$, тогда в текущий момент времени $t=t_{k}$ функция $u\left(t_{k}, \rho\right)$ является обратимой. Для обратной к ней функции $\rho=\rho\left(t_{k}, u\right)$ уравнение (2.1) примет вид:

$$
\rho_{t} \rho_{u}^{2}=u \rho_{u u}-\frac{\rho_{u}}{\sigma}-\frac{v u \rho_{u}^{2}}{\rho}
$$

Граничное условие (2.3) можно записать следующим образом:

$$
\left.\rho\right|_{u=f(t)}=R
$$

Таким образом, решение задачи (2.1) и (2.3) в каждый момент времени будет обратной функцией к решению задачи (5.1) и (5.2). Решение последней в свою очередь на каждом шаге по времени $t=t_{k}$ можно свести к решению в известной области $W_{k}: u \in[0, L]$, $L=f\left(t_{k}\right)$, следующей краевой задачи:

$$
\begin{gathered}
\rho_{u u}=\frac{1}{u}\left(\rho_{t} \rho_{u}^{2}+\frac{\rho_{u}}{\sigma}\right)+\frac{v \rho_{u}^{2}}{\rho} ; \\
\left.\rho\right|_{u=L}=R .
\end{gathered}
$$

Неизвестный нулевой фронт для исходной задачи соответствует значениям искомой функции $\rho\left(t_{k}, u\right)$ при $u=0$ :

$$
\left.\rho\right|_{u=0}=a\left(t_{k}\right) .
$$

Из условия (4.3) следует:

$$
\left.q^{(\rho)}\right|_{u=0}=\left.\frac{\partial \rho}{\partial n}\right|_{u=0}=\frac{1}{\sigma a^{\prime}\left(t_{k}\right)} .
$$

Здесь $q^{(\rho)}-$ поток для функции $\rho(t, u)$. Итерационный алгоритм решения задачи $(5.3)$, (5.4) и (5.6) методом граничных элементов, аналогичный описанным в предыдущем разделе алгоритмам решения одномерных задач о движении теплового фронта, позволит найти 
на каждом шаге по времени непрерывную функцию $\rho=\rho\left(t_{k}, u\right)$, обратную к решению исходной задачи. Непрерывность обеспечивает обращение без потери точности.

Вычислительный эксперимент показал устойчивую сходимость итерационных процедур и повышение точности решения задач об инициировании волны по сравнению с решениями в исходных обозначениях, описанными в разд. 4.

Рассмотрим теперь двумерную задачу об инициировании тепловой волны в полярных координатах (2.7) и (2.9). В момент времени $t=t_{k}$ область решения задачи (область ненулевых значений искомой функции $\left.u\left(t_{k}, \rho, \varphi\right)\right)$ ограничена линией $\rho=R(\varphi)$ и нулевым фронтом - неизвестной линией $\rho=b\left(t_{k}, \varphi\right)$. Отметим, что $b(0, \varphi)=R(\varphi)$. Поменяем местами в задаче (2.7) и (2.9) искомую функцию $u$ и координату $\rho$, а затем сделаем замену переменной $v=u+1$. Уравнение (2.7) для новой искомой функции $\rho\left(t_{k}, v, \varphi\right)$ примет следующий вид:

$$
\rho_{t} \rho_{v}^{2}=(v-1)\left(\rho_{v v}-\frac{\rho_{v}^{2}}{\rho}-\frac{\rho_{v}\left(\rho_{v \varphi} \rho_{\varphi}-\rho_{\varphi \varphi} \rho_{v}\right)}{\rho^{2}}\right)-\frac{1}{\sigma}\left(\rho_{v}+\frac{\rho_{v} \rho_{\varphi}^{2}}{\rho^{2}}\right) .
$$

Будем рассматривать далее переменные $v, \varphi$ как полярные координаты в плоскости декартовых координат $\xi, \eta: \xi=v \cos \varphi, \eta=v \sin \varphi$. Краевое условие (2.9) примет на шаге $t=t_{k}$ вид

$$
\left.\rho\right|_{v=1+f\left(t_{k}, \varphi\right)}=R(\varphi)
$$

Условие $v=1+f\left(t_{k}, \varphi\right)$ задает в плоскости координат $\xi$, $\eta$ в каждый момент времени замкнутую линию $C^{\left(t_{k}\right)}$, ограничивающую область $U^{\left(t_{k}\right)}$, содержащую начало координат, при этом $C^{(0)}$ - окружность единичного радиуса с центром в начале координат.

Решению задачи (2.7) и (2.9) на шаге $t=t_{k}$ в новых переменных соответствует задача (5.7) и (5.8), состоящая в определении функции $\rho=\rho\left(t_{k}, v, \varphi\right),(v, \varphi) \in W^{\left(t_{k}\right)}$, где $W^{\left(t_{k}\right)}-$ известная в каждый момент область, ограниченная линиями $C^{(0)}$ и $C^{\left(t_{k}\right)}$. В свою очередь задача (5.7) и (5.8) сводится к краевой задаче в области $W^{\left(t_{k}\right)}$ для уравнения Пуассона

$$
\Delta \rho=\frac{1}{v-1}\left(\rho_{t} \rho_{v}^{2}+\frac{1}{\sigma}\left(\rho_{v}+\frac{\rho_{v} \rho_{\varphi}^{2}}{\rho^{2}}\right)\right)+\frac{\rho_{v}}{v}+\frac{\rho_{\varphi \varphi}}{v^{2}}+\frac{\rho_{v}^{2}}{\rho}+\frac{\rho_{v}\left(\rho_{v \varphi} \rho_{\varphi}-\rho_{\varphi \varphi} \rho_{v}\right)}{\rho^{2}}
$$

с граничными условиями (5.8) и

$$
\left.q^{(\rho)}\right|_{\nu=1}=\left.\frac{\partial \rho}{\partial n}\right|_{\nu=1}=\frac{1}{\sigma \rho_{t}}\left(1+\frac{\rho_{\varphi}^{2}}{\rho^{2}}\right) .
$$

Итерационное решение задачи (5.9), (5.8) и (5.10) методом граничных элементов, аналогично решению двумерной задачи о движении теплового фронта, описанному в предыдущем разделе, позволит определить на каждом шаге $t=t_{k}$ непрерывную по пространственным переменным функцию $\rho=\rho\left(t_{k}, v, \varphi\right)$, обратную к решению исходной задачи. 
Таким образом, преобразование переменных позволило построить алгоритм численного решения двумерной задачи об инициировании тепловой волны с помощью метода граничных элементов.

\section{6. Частично-автомодельные решения}

Важным этапом всякого численного исследования нелинейных уравнений математической физики является проверка корректности результатов вычислительного эксперимента. Одним из наиболее простых и эффективных способов этого является сравнение последних с точными решениями. Будем искать решения задачи (2.1) и (2.2) в случае круговой симметрии $(v=1)$ в виде

$$
u=\lambda(t) w(z), z=1-\frac{\rho}{a(t)}
$$

Решения подобного вида будем называть частично-автомодельными. Подставив (6.1) в уравнение, после приведения подобных и умножения обеих частей на $a^{2}(t) / \lambda^{2}(t)$ получим

$$
w w^{\prime \prime}+\frac{1}{\sigma}\left(w^{\prime}\right)^{2}+\left[\frac{a(t) a^{\prime}(t)}{\lambda(t)}(z-1)+\frac{w}{z-1}\right] w^{\prime}-\frac{a^{2}(t) \lambda^{\prime}(t)}{\lambda^{2}(t)} w=0 .
$$

Утверждение 1. Уравнение (6.2) является относительно $w(z)$ обыкновенным дифференциальным тогда и только тогда, когда закон движения фронта тепловой волнь а $(t)$ является либо показательной, либо степенной функичией.

Доказательство. Можно видеть, что (6.2) будет обыкновенным дифференциальным уравнением (ОДУ), в котором искомой функцией является $w(z)$, тогда и только тогда, когда выполнены равенства

$$
\frac{a(t) a^{\prime}(t)}{\lambda(t)}=A=\text { const }, \frac{a^{2}(t) \lambda^{\prime}(t)}{\lambda^{2}(t)}=B=\text { const } .
$$

Проинтегрировав систему (6.3), можно убедиться, что, если $A / B^{2}=2$, решением является показательная функция $a(t)=C_{1} e^{C_{2} z}$; если же $A / B^{2} \neq 2$ - степенная функция $a(t)=\left(C_{3} t+C_{4}\right)^{\omega}$. Здесь $C_{i}, i=1, \ldots, 4 ; \omega-$ константы. Утверждение доказано.

При выполнении условий утверждения 1 уравнение (6.2) может быть записано в виде

$$
w w^{\prime \prime}+\frac{1}{\sigma}\left(w^{\prime}\right)^{2}+\left[z-1+\frac{w}{z-1}\right] w^{\prime}-\eta w=0,
$$

где $\eta=2$ в случае показательного закона движения фронта тепловой волны; $\eta=(2 \omega-1) / \omega$ в случае степенного закона движения. Условие (2.2) переходит в

$$
w(0)=0
$$

В соответствии с физическим смыслом задачи будем требовать, чтобы $w^{\prime}(0)>0$. Тогда условием совместности уравнения (6.4) является равенство 


$$
w^{\prime}(0)=\sigma
$$

Существование и единственность аналитического решения задачи Коши (6.4)-(6.6) следует из ранее полученных результатов авторов [10].

Таким образом, получены следующие частично-автомодельные решения задачи (2.1) и (2.2) в случае круговой симметрии $(v=1)$ :

$$
u=\left\{\begin{array}{c}
C_{1}^{2} C_{2} e^{2 C_{2} t} w(z), z=1-\frac{\rho}{C_{1} e^{C_{2} t}}, \\
C_{3} \omega\left(C_{3} t+C_{4}\right)^{2 \omega-1} w(z), z=1-\frac{\rho}{\left(C_{3} t+C_{4}\right)^{\omega}} .
\end{array}\right.
$$

Здесь функция $w(z)$ удовлетворяет задаче Коши (6.4)-(6.6) с соответствующим значением параметра $\eta$.

При некоторых значениях входящих констант решение задачи (6.4)-(6.6) можно получить в квадратурах и, соответственно, записать (6.7) в виде конечной формулы. Построенные частично-автомодельные решения используются для верификации расчетов в следующем разделе.

\section{7. Примеры}

В качестве первого примера рассмотрим задачу о движении теплового фронта в случае круговой симметрии, когда закон движения фронта тепловой волны задан показательной функцией:

$$
\begin{gathered}
u_{t}=u u_{\rho \rho}+\frac{1}{\sigma} u_{\rho}^{2}+\frac{1}{\rho} u u_{\rho} ; \\
\left.u\right|_{\rho=e^{t}}=0 .
\end{gathered}
$$

Задача (7.1) и (7.2) была решена методом граничных элементов с помощью алгоритма, описанного в разд. 4. Решение было построено на интервале $t \in[0,1]$ с шагом $h=0,01$ при $\sigma=3$. Для верификации решения было использовано частично-автомодельное решение

$$
u(t, \rho)=e^{2 t} w(z), z=1-\frac{\rho}{e^{t}},
$$

где $w(z)$ - решение соответствующей задачи (6.4)-(6.6).

На рис. 1 показано сравнение полученных решений, демонстрирующее высокую степень их близости.

Аналогичное сравнение было проведено при краевом условии, соответствующем линейному закону движения теплового фронта:

$$
\left.u\right|_{\rho=t+1}=0 .
$$

Показанные на рис. 2 решение МГЭ и частично-автомодельное решение также достаточно близки. 


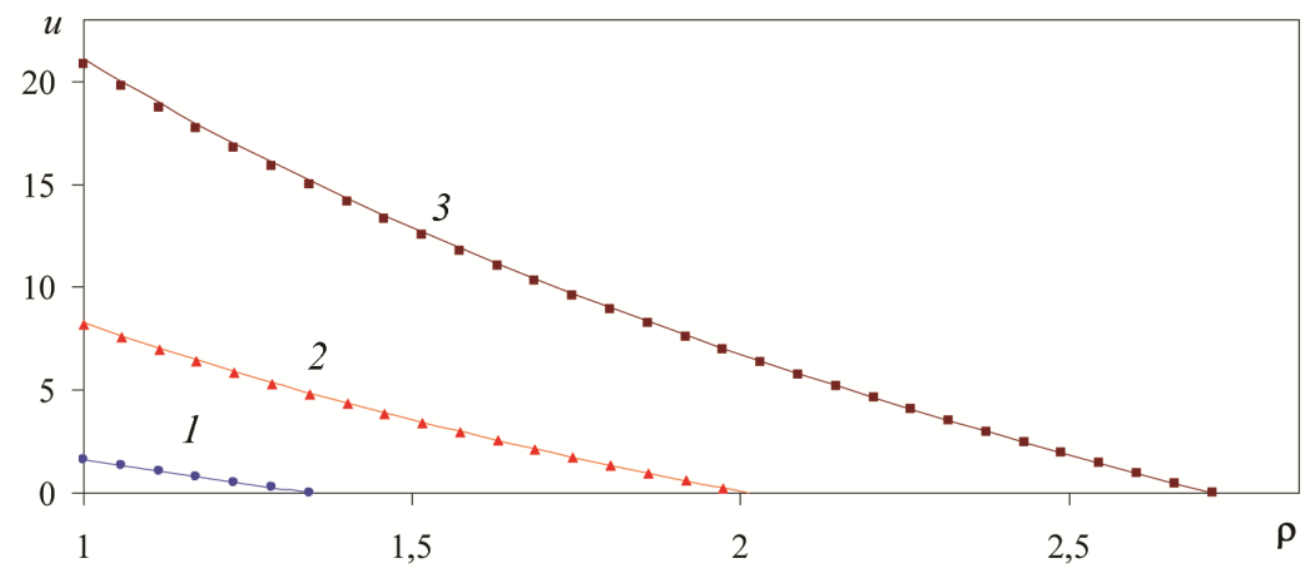

Рис. 1. Сравнение решения МГЭ и частично-автомодельного решения для показательного закона движения фронта тепловой волны: $1-t=0,3 ; 2-t=0,7 ; 3-t=1$

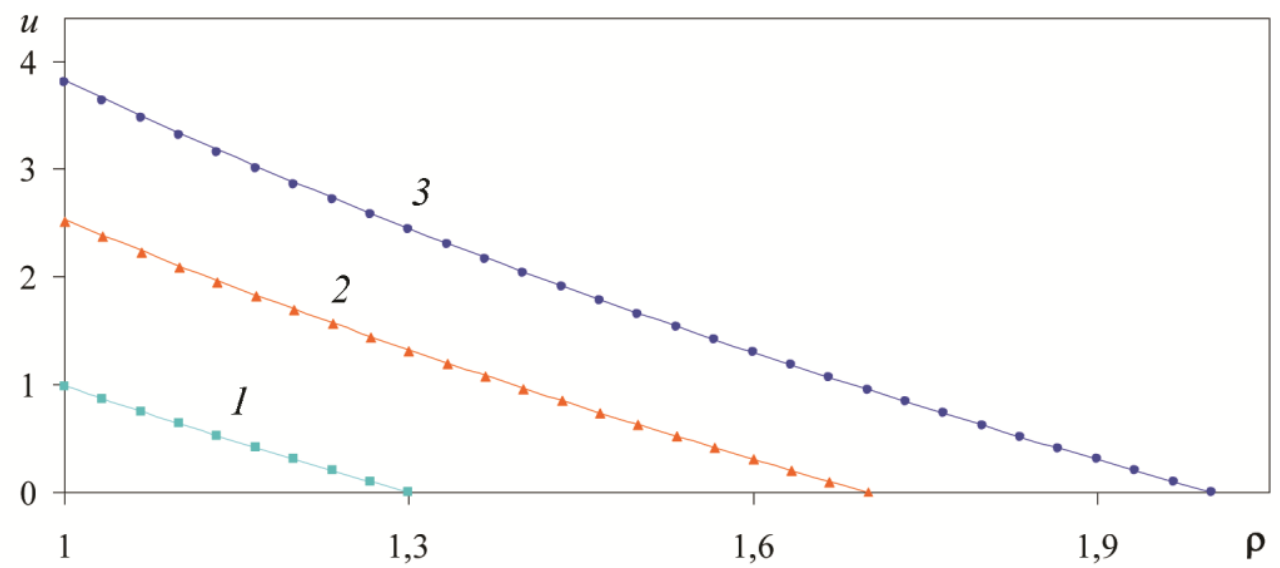

Рис. 2. Сравнение решения МГЭ и частично-автомодельного решения для линейного закона движения фронта тепловой волны: $1-t=0,3 ; 2-t=0,7 ; 3-t=1$

Проведенные расчеты показали, что построение частично-автомодельных решений является эффективным средством верификации численных решений задач для нелинейного уравнения теплопроводности, а также еще раз дали возможность оценить точность решений на основе МГЭ.

\section{8. Заключение}

В статье приведен обзор методов и результатов, полученных авторами в ходе исследования решений нелинейного уравнения теплопроводности, имеющих тип тепловой волны, распространяющейся по холодному (нулевому) фону с конечной скоростью. Тепловые волны обладают особенностью на фронте, поскольку там обращается в нуль множитель перед старшей производной, вследствие чего обычные численные методы оказываются неприменимыми. Авторами для построения решений обычно используются метод степенных рядов, позволяющий раскрыть особенность и построить локальное решение (в малом по $t$ ), и метод граничных элементов, с использованием которого на заданном конечном промежутке времени строятся численно-аналитические решения. В работе проведен сравнительный анализ указанных подходов, который показал, что метод граничных элементов несколько сложнее в реализации и для него труднее доказать какие-либо строгие факты о сходимости (на данный момент этого сделать не удалось), однако он позволяет строить решения на более продолжительном временном интервале. Также рассмотрены частные случаи, когда решение задачи о движении тепловой волны сводится к задаче Коши для обыкновенного дифференциального 
уравнения. Такие решения могут быть использованы для построения точных решений и для верификации численных расчетов. Дальнейшие исследования по тематике статьи могут быть связаны с разработкой методов исследования получаемых задач Коши, построением новых частично-автомодельных решений, а также с увеличением размерности задач, решаемых методом граничных элементов, с двух до трех.

\section{Благодарность}

Работа выполнена при частичной поддержке Комплексной программь УрО РАН, проект № 18-1-1-5, и РФФИ, проект № 16-01-00608.

\section{Литература}

1. Vazquez J. L. The Porous Medium Equation: Mathematical Theory. - Oxford : Clarendon Press, 2007. - 648 p. - ISBN-10: 0198569033, ISBN-13: 978-019856903.

2. Режимы с обострением в задачах для нелинейных параболических уравнений / А. А. Самарский, В. А. Галактионов, С. П. Курдюмов, А. П. Михайлов - М. : Наука, 1987. $476 \mathrm{c}$.

3. Зельдович Я. Б., Компанеец А. С. К теории распространения тепла при теплопроводности, зависящей от температуры // В кн.: Сборник, посвященный 70-летию академика А. Ф. Иоффе. - М. : Изд-во АН СССР, 1950. - С. 61-71.

4. Баренблатт Г. И., Вишик И. М. О конечной скорости распространения в задачах нестационарной фильтрации жидкости и газа // Прикладная математика и механика. - 1956. Т. 20, вып. 3. - С. 411-417.

5. Олейник О. А., Калашников А. С., Чжоу Юй-Линь. Задача Коши и краевые задачи для уравнений типа нестационарной фильтрации // Известия АН СССР. Серия математическая. - 1958. - Т. 22, вып. 5. - С. 667-704.

6. Сидоров А. Ф. Избранные труды: Математика. Механика. - М. : Физматлит, 2001. 576 c. - ISBN 5-9221-0103-X.

7. Полянин А. Д., Зайцев В. Ф., Журов А. И. Нелинейные уравнения математической физики и механики. Методы решения. - М. : Изд-во Юрайт, 2017. - 256 с. - ISBN 978-5-534-02317-6.

8. Казаков А. Л., Лемперт А. А. Аналитическое и численное исследование одной краевой задачи нелинейной фильтрации с вырождением // Вычислительные технологии. - 2012. T. 17, № 1. - С. 57-68.

9. Kazakov A. L., Lempert A. A. Existence and Uniqueness of the Solution of the Boundary-Value Problem for a Parabolic Equation of Unsteady Filtration. Journal of Applied Mechanics and Technical Physic - 2013. - Vol. 54, iss. 2. - P. 251-258. - URL: https://doi.org/10.1134/S0021894413020107.

10. Казаков А. Л., Кузнецов П. А. Об аналитических решениях одной специальной краевой задачи для нелинейного уравнения теплопроводности в полярных координатах // Сибирский журнал индустриальной математики. - 2018. - Т. 21, № 2(74). - С. 56-65. DOI: 10.17377/SIBJIM.2018.21.205

11. Казаков А. Л., Кузнецов П. А., Спевак Л. Ф. Об одной краевой задаче с вырождением для нелинейного уравнения теплопроводности в сферических координатах // Труды Института математики и механики УрО РАН. - 2014. - Т. 20, № 1. - С. 119-129.

12. Казаков А. Л., Спевак Л. Ф. Методы граничных элементов и степенных рядов в одномерных задачах нелинейной фильтрации // Известия ИГУ. Серия «Математика». - 2012. T. 5, № 2. - C. 2-17.

13. Kazakov A. L., Spevak L. F. Numerical and analytical studies of a nonlinear parabolic equation with boundary conditions of a special form // Applied Mathematical Modelling. - 2013. Vol. 37, iss. 10-11. - P. 6918-6928. - DOI: 10.1016/j.apm.2013.02.026 
14. Kazakov A. L., Spevak L. F. An analytical and numerical study of a nonlinear parabolic equation with degeneration for the cases of circular and spherical symmetry // Applied Mathematical Modelling. 2015. - Vol. 40, iss. 2. - P. 1333-1343. - DOI: 10.1016/j.apm.2015.06.038

15. Spevak L. F., Nefedova O. A. Solving a two-dimensional nonlinear heat conduction equation with degeneration by the boundary element method with the application of the dual reciprocity method // AIP Conference Proceedings. - 2016. - Vol. 1785, iss. 1. - P. 040077. URL: http://doi.org/10.1063/1.4967134

16. Казаков А. Л., Спевак Л. Ф., Нефедова О. А. Решение двумерной задачи о движении фронта тепловой волны с использование степенных рядов и метода граничных элементов // Известия ИГУ. Серия «Математика». - 2016. - Т. 18. - С. 21-37.

17. Banerjee P. K., Butterheld R. Boundary element methods in engineering science. US : McGraw-Hill Inc., 1981. - 452 p. ISBN-10: 0070841209, ISBN-13: 978-0070841208.

18. Brebbia C. A., Telles J. F. C., Wrobel L. C. Boundary Element Techniques. - Berlin, Neidelberg, New-York, Tokyo: Springer-Verlag, 1984. - 466 p. - ISBN 978-3-642-48862-7. - DOI: 10.1007/978-3-642-48860-3

19. Nardini D., Brebbia C. A. A New Approach to Free Vibration Analysis using Boundary Elements // Applied Mathematical Modelling. - 1983. - Vol. 7, no. 3. - P. 157-162. DOI: 10.1016/0307-904X(83)90003-3

20. Fedotov V. P., Spevak L. F. One approach to the derivation of exact integration formulae in the boundary element method // Engineering Analysis with Boundary Elements. - 2008. - Vol. 32, no. 10. - P. 883-888. - DOI: $10.1016 /$ j.enganabound.2008.03.001 